SRC TR $85-4$

A New Parameter Estimate in Singular Perturbations

by

E.H. Abed 
A New Parameter Estimate in Singular Perturbations

by

Eyad H. Abed 


\title{
A New Parameter Estimate in Singular Perturbations
}

\author{
Eyad H. Abed \\ Department of Electrical Engineering \\ University of Maryland, College Park, MD 20742
}

\begin{abstract}
A new upper bound is obtained for the singular perturbation parameter of an asymptotically stable singularly perturbed system. General time-invariant systems with a single small parameter are considered. The paper employs a Riccati equation whose solution is known to facilitate the exact decoupling of fast and slow dynamics. An application of the Brouwer fixed point theorem to the Riccati equation and of Liapunov's direct method to the fast and slow subsystems results in the desired upper bound. Computation of the estimate requires only the solution of two Liapunov matrix equations. For the case of scalar fast and slow variables, an expression for the estimate is evaluated and compared with the upper bound of Zien (L. Zien, J. Franklin Institute, 295, pp. 373-381, 1973).
\end{abstract}

Keywords: Singular perturbation, Robustness of stability, Asymptotic stability, Fixed point methods, Liapunov's direct method. 


\section{Introduction}

Consider the system

$$
\dot{x}=A x+B y
$$

$$
\epsilon \dot{y}=C x+D y
$$

in which $\epsilon>0$ is a small real parameter, $x$ and $y$ are vectors in $R^{n}$ and $R^{m}$ respectively, and $A, B, C, D$ are matrices of appropriate dimension. System (1) is referred to as a singularly perturbed system since its dimension drops from $n+m$ to $n$ when the parameter $\epsilon$ is formally set to 0. Theorem 1 below gives sufficient conditions for the asymptotic stability of (1) for all sufficiently small values of the singular perturbation parameter $\epsilon$ and has been derived by several authors. See the excellent survey articles $[17,18,19]$ for a discussion of results in singular perturbation theory and for references to the early literature.

Notation. By ' $a:=b$ ' one intends that $a$ is defined as $b$. For a square matrix $G, \sigma(G)$ denotes the spectrum (set of eigenvalues) of $G$. Throughout the paper the spectral (or $L_{2}$ ) norm is used and is indicated by $\mid$. This is for simplicity and does not represent a limitation of the method. (Recall that $|P|^{2}=\lambda_{\max }\left(P^{\prime} P\right)$ for any real matrix $P$, where $\lambda_{\max }\left(P^{\prime} P\right)$ denotes the largest eigenvalue of $P^{\prime} P$; If $\mathrm{P}$ is a vector, this is the Euclidean norm of $\left.\mathrm{P}[21].\right)$ Finally, $J(\epsilon)$ will denote the Jacobian matrix

$$
J(\epsilon):=\left(\begin{array}{cc}
A & B \\
\epsilon^{-1} C & \epsilon^{-1} D
\end{array}\right)
$$

of (1) at the equilibrium $(x, y)=(0,0)$.

Recall that a matrix is a stability matrix (or simply stable) if its spectrum lies in the open left half complex plane [3].

Theorem 1 [11]. Let $D$ be nonsingular. If the matrices $A_{0}:=A-B D^{-1} C$ and $D$ are stable, then there exists an $\bar{\epsilon}>0$ such that the null solution of system (1) is asymptotically stable for all $\epsilon \in(0, \bar{\epsilon})$.

Since the parameter $\epsilon$ typically represents a small physical quantity over which one has little or no control, it is of practical significance to find explicit upper bounds $\epsilon_{0}$ on $\epsilon$ ensuring that the conclusion of the theorem above is valid for all $\epsilon \in\left(0, \epsilon_{0}\right)$. The aim of this paper is to derive one such upper bound. The derivation consists of two main steps. First, the Brouwer fixed point theorem is used to obtain an initial upper bound on $\epsilon$ which ensures the existence of a solution to an associated algebraic matrix Riccati equation. This initial upper bound is parametrized by the magnitude of a norm constraint imposed on a solution to the Riccati equation. The referenced Riccati equation (Eq. (6) below) is useful since its solution(s) can be used to produce a nonsingular similarity transformation whose effect is to explicitly separate the fast and slow dynamics of (1). Next, Liapunov's direct method is applied to obtain a bound on the norm of the solution to the Riccati equation which ensures asymptotic stability of the fast and slow subsytems. An explicit upper bound on the singular perturbation parameter $\epsilon$ readily follows.

It appears that Zien's 1973 paper [23] was the first to obtain concrete results on this issue. More recently, Javid [13] derived an upper bound valid for linear time-varying systems. Since [23] and [13] used similar contraction mapping arguments on function space, it is not surprising that the upper bound of [13] reduces to that of [23] in the time-invariant case. Sandell [20] has studied the problem using the singular value decomposition, obtaining an inequality involving $\epsilon$ which was then used to yield explicit upper bounds in specific examples, though no generally applicable formula for an upper bound was derived. Chow [7] contains a stability computation for a class of nonlinear autonomous singularly perturbed systems which can be directly applied to 
yield an explicit upper bound for $\epsilon$. Finally, Khalil [15] studies a class of nonlinear systems in which several singular perturbation parameters $\epsilon_{1}, \ldots, \epsilon_{M}$ may appear. An upper bound on $\left(\epsilon_{1} \epsilon_{2} \cdots \epsilon_{M}\right)^{1 / M}$ is obtained by a Liapunov function argument under the assumption that the mutual ratios $\epsilon_{i} / \epsilon_{j}$ are bounded from above and below by known positive constants.

\section{Separation of Fast and Slow Modes}

It is well known $[6,16,19,18,8,1]$ that if $D$ is nonsingular the Riccati equation for the $m \times n$ matrix $L$

$$
D L-\epsilon L A+\epsilon L B L-C=0
$$

can be used to exactly separate the fast and slow dynamics of (1) for those values of $\epsilon$ for which (3) has a solution. Moreover, one has the following representation for the spectrum of $J(\epsilon)$;

$$
\sigma(J(\epsilon))=\sigma(A-B L) \bigcup \sigma\left(\epsilon^{-1} D+L B\right) .
$$

If $L$ exists, (4) implies that the eigenvalues of $(A-B L)$ are the slow modes and those of $\left(\epsilon^{-1} D+L B\right)$ are the fast modes of (1). In this paper Eq. (4) is all that is required from the results on explicit separation of fast and slow modes and the reader is referred to the survey articles mentioned above for further details. Note that by Eq. (3) $L=D^{-1} C+O$ ( $|\epsilon|$ ). It will prove convenient in the computations below to use the matrix

$$
\Gamma:=D^{-1} C-L
$$

in favor of $L$, as $\Gamma=O(|\epsilon|)$. In terms of $\Gamma$, (3) becomes [1]

$$
D \Gamma+\epsilon\left(D^{-1} C-\Gamma\right) A_{0}-\epsilon \Gamma B \Gamma+\epsilon D^{-1} C B \Gamma=0,
$$

where $A_{0}=A-B D^{-1} C$ is as defined in Theorem 1.

Rewrite Eq. (6) in the fixed point form

$$
F_{\epsilon}(\Gamma)=\Gamma
$$

where the parametrized mapping $F_{\epsilon}: R^{m \times n} \rightarrow R^{m \times n}$ is defined for any real $\epsilon$ by

$$
F_{\epsilon}(\Gamma):=\epsilon D^{-1}\left\{\left(\Gamma-D^{-1} C\right) A_{0}+\Gamma B \Gamma-D^{-1} C B \Gamma\right\} .
$$

A parametrized upper bound $\epsilon_{1}(\alpha)$ on $\epsilon$ will now be derived such that for any $\alpha>0$ and for all $\epsilon \in\left(0, \epsilon_{1}(\alpha)\right],|\Gamma| \leq \alpha$ implies $\left|F_{\epsilon}(\Gamma)\right| \leq \alpha$. Proceeding, let $\alpha>0$ be given and let $\Gamma \in R^{m \times n}$ satisfy $|\Gamma| \leq \alpha$. From (8) one has

$$
\begin{aligned}
\left|F_{\epsilon}(\Gamma)\right| & \leq \epsilon\left|D^{-1}\right|\left\{\left(|\Gamma|+\left|D^{-1} C\right|\right)\left|A_{0}\right|+|B||\Gamma|^{2}+\left|D^{-1} C B\right||\Gamma|\right\} \\
& \leq \epsilon\left|D^{-1}\right|\left\{\left(\alpha+\left|D^{-1} C\right|\right)\left|A_{o}\right|+\alpha^{2}|B|+\alpha\left|D^{-1} C B\right|\right\} .
\end{aligned}
$$

From (9) follows immediately that for $\left|F_{\epsilon}(\Gamma)\right| \leq \alpha$ to hold it suffices that $\epsilon \leq \epsilon_{\mathrm{l}}(\alpha)$ where $\epsilon_{1}(\alpha)$ is given by

$$
\epsilon_{1}(\alpha):=\frac{\alpha}{\left|D^{-1}\right|\left\{\left|D^{-1} C\right|\left|A_{0}\right|+\alpha\left(\left|D^{-1} C B\right|+\left|A_{0}\right|\right)+\alpha^{2}|B|\right\}} .
$$

Thus for any $\alpha>0, F_{\epsilon}$ is a continuous map of the closed ball $B_{\alpha}:=\left\{\Gamma \in R^{m \times n}:|\Gamma| \leq \alpha\right\}$ into itself whenever $0 \leq \epsilon \leq \epsilon_{1}(\alpha)$. Note that $B_{\alpha}$ is homeomorphic to the closed unit ball in $R^{m n}$. The Brouwer fixed point theorem ( $[4]$, p. 54 and [12], p. 10 ) may now be invoked to establish the existence of a solution $\Gamma(\epsilon)$ to $(7)$ in $B_{\alpha}$ for any $\epsilon \in\left(0, \epsilon_{1}(\alpha)\right]$.

Theorem 2. For any $\alpha>0$ and for all $\epsilon \leq \epsilon_{1}(\alpha)$, the Riccati equation (6) has at least one solution $\Gamma(\epsilon)$ with $|\Gamma(\epsilon)| \leq \alpha$.

Remark 1. Note that for any system (1) with $B \neq 0$ (the generic and nontrivial case), the upper bound $\epsilon_{\mathrm{I}}(\alpha)$ approaches 0 as $\alpha \rightarrow \infty$. It is clear, however, that a solution of (6) in the ball $B_{\alpha}$ for a given $\alpha=\alpha_{0}$ will also belong to any ball $B_{\alpha}$ with $\alpha>\alpha_{0}$. Therefore the upper bound of 
Theorem 2 can be made less conservative by using instead of $\epsilon_{1}(\alpha)$ the revised estimate $\epsilon_{1}^{*}(\alpha):=\max _{0 \leq \beta \leq \alpha} \epsilon_{1}(\beta)$.

Remark 2. It is natural to attempt to factorize the quadratic appearing in the denominator of the expression (10) for $\epsilon_{1}(\alpha)$. In this regard, note that replacing $\left|D^{-1} C B\right|$ in (10) by $\left|D^{-1} C\right||B|$ results in the new (in general more conservative) upper bound $\epsilon_{1}^{* *}$ given by

$$
\epsilon_{1}^{* *}(\alpha):=\frac{\alpha}{\left|D^{-1}\right|\left(\left|D^{-1} G\right|+\alpha\right)\left(\left|A_{0}\right|+\alpha|B|\right)} .
$$

Remark 3. Note that in the derivation of Theorem 2 only existence of a solution $\Gamma$ to (6) needed to be ensured. A further upper bound on $\epsilon$ to ensure uniqueness could easily be derived by a contraction mapping argument as in ( $[8]$, pp. 16-18). However, this could only result in a more conservative final estimate and would thus be counterproductive.

\section{Perturbational Stability Analysis}

\subsection{A basic perturbational result}

The point of departure of this section is Brockett's proof of a basic perturbational result on stability of linear systems (Brockett [5], p. 205). In [5] one is concerned with the persistence of exponential stability of time varying linear systems under sufficiently small linear perturbations. The specialization of this result to the time-invariant case is elementary and is easily stated.

Proposition 1. Let $F$ be a constant square matrix and suppose that $\dot{x}=F x$ is asymptotically stable. Then there is a $\mu>0$ such that if $\Delta$ is a constant matrix with $|\Delta|<\mu$, then $\dot{x}=(F+\Delta) x$ is also asymptotically stable.

Note that Proposition 1 follows from continuity of the eigenvalues of a matrix in its parameters (Kato [14], p. 107). The proof of the time varying version of Proposition 1 in [5] will now be specialized to yield an explicit upper bound $\mu^{*}$ for $|\Delta|$.

Since $F$ is stable there is a unique, positive definite matrix $Q$ which solves the Liapunov matrix equation $F^{\prime} Q+Q F=-I[5,22]$. Moreover, $Q$ is given by the convergent integral

$$
Q=\int_{0}^{\infty} e^{F^{\prime} t} e^{F t} d t
$$

Using $x^{\prime} Q x$ as a candidate Liapunov function for the perturbed system $\dot{x}=(F+\Delta) x$, one computes

$$
\begin{gathered}
\frac{d}{d t} x^{\prime} Q x=x^{\prime}\left(F^{\prime} Q+Q F+Q \Delta+\Delta^{\prime} Q\right) x \\
=-x^{\prime} x+x^{\prime}\left(Q \Delta+\Delta^{\prime} Q\right) x
\end{gathered}
$$

Noting that there is a $\mu$ such that $|\Delta|<\mu$ implies

$$
\left|Q \Delta+\Delta^{\prime} Q\right|<1
$$

completes the proof of Proposition 1.

To obtain an explicit upper bound $\mu^{*}$, use the triangle inequality : $\left|Q \Delta+\Delta^{\prime} Q\right| \leq|Q|\left(|\Delta|+\left|\Delta^{\prime}\right|\right)=2|Q||\Delta|$. (The last equality follows from the fact that the spectral norm of a matrix equals that of its transpose [21].) Eq. (14) now yields the following result. 
Proposition 2. Let the assumptions of Proposition 1 hold. Then $F+\Delta$ will be a stability matrix if $|\Delta|<\mu^{*}$ where

$$
\mu^{*}:=\frac{1}{2|Q|}
$$

and where $Q$ is given by Eq. (12).

\subsection{Stability of the slow subsystem}

As noted earlier, (4) implies that the eigenvalues of the separated slow subsystem are precisely the eigenvalues of the matrix $(A-B L)=$ : $A^{6}$ if a solution $L$ to (3) exists. In terms of $\Gamma$ (see Eq. (5)) this matrix is given by

$$
\begin{aligned}
A^{8} & =A-B D^{-1} C+B \Gamma \\
& =A_{0}+B \Gamma,
\end{aligned}
$$

with $\Gamma$ a solution to (6). Proposition 2 now implies that $A^{*}$ is a stability matrix (i.e. the slow modes are stable) if (i) $A_{0}$ is stable, and (ii) there is a solution $\Gamma$ of (6) with $|B \Gamma|<\frac{1}{2\left|Q^{8}\right|}$ where

$$
Q^{\bullet}:=\int_{0}^{\infty} e^{A_{0}^{\prime} t} e^{A_{0} t} d t .
$$

This will be true if the hypotheses of Theorem 1 hold and if

$$
|\Gamma|<\frac{1}{2|B|\left|Q^{*}\right|}
$$

is satisfled, where $\Gamma$ is some solution to Eq. (6).

\subsection{Stability of the fast subsystem}

By Eq. (4) and the definition (5) of $\Gamma$, the eigenvalues of the fast subsystem are those of the matrix $\epsilon^{-1} D+D^{-1} C B-\Gamma B$. Since $\epsilon>0$ the fast subsystem is stable if and only if the matrix

$$
A^{\prime}:=D+\epsilon D^{-1} C B-\epsilon \Gamma B
$$

is stable. By Proposition 2, this will be the case if (i) D is stable, and (ii) the inequality

$$
\left|\epsilon D^{-1} C B-\epsilon \Gamma B\right|<\frac{1}{2\left|Q^{f}\right|}
$$

is valid, where $Q^{f}$ is defined as

$$
Q^{f}:=\int_{0}^{\infty} e^{D^{\prime} t} e^{D t} d t
$$

By the triangle inequality and Eq. (18), the left side of (20) is not larger than $\epsilon\left(\left|D^{-1} C B\right|+\frac{1}{2\left|Q^{8}\right|}\right)$. Deffining

$$
\epsilon_{2}:=\frac{\left|Q^{*}\right|}{\left|Q^{\prime}\right|\left(1+2\left|Q^{*}\right|\left|D^{-1} C B\right|\right)},
$$

it now follows that the fast subsystem is stable if (i) a solution $\Gamma$ to (6) exists which satisfles the bound (18), and (ii) $\epsilon<\epsilon_{2}$. 


\section{The Upper Bound}

It is now straightforward to give an upper bound $\epsilon_{0}$ on $\epsilon$ such that for $0<\epsilon<\epsilon_{0}$ the asymptotic stability of $(1)$ is certain. Let the hypotheses of Theorem 1 be satisfied. By the results of Section 3.2 , the slow subsystem is well defined and its stability is ensured if there is a solution $|\Gamma|$ of Eq. (6) satisfying the inequality (18). By Theorem 2, this will be the case if $\epsilon \leq \epsilon_{1}(\alpha)$ where $\alpha$ is any positive number satisfying

$$
\alpha<\frac{1}{2|B|\left|Q^{8}\right|}=: \bar{\alpha} \text {. }
$$

Finally, the fast subsystem will be well defined and stable if the last remark holds and if $\epsilon<\epsilon_{2}$ where $\epsilon_{2}$ is given by Eq. (22). By Eq. (4) stability of the fast and slow subsystems implies that of the singularly perturbed system (1).

These remarks are summarized in the following theorem, in which the upper bound $\epsilon_{0}$ of this paper is stated explicitly. Note that the freedom in $\alpha$ is reflected in taking a maximum over $0 \leq \alpha \leq \bar{\alpha}$ in Eq. (24).

Theorem 3. Let the assumptions of Theorem 1 hold, so that both $A_{0}=A-B D^{-1} C$ and $D$ are stability matrices. Then the null solution of (1) is asymptotically stable for all $\epsilon$ in the open interval $\left(0, \epsilon_{0}\right)$ where $\epsilon_{0}$ is given by

$$
\epsilon_{0}:=\min \left(\max _{0 \leq \alpha \leq \bar{\alpha}} \epsilon_{1}(\alpha), \epsilon_{2}\right)
$$

and where $\epsilon_{1}(\alpha), \epsilon_{2}$ and $\bar{\alpha}$ are given by Eqs. (10), (22) and (23) respectively.

Remark 4. Note that $\epsilon_{0}$ is simply min $\left(\epsilon_{1}^{*}(\bar{\alpha}), \epsilon_{2}\right)$ where $\epsilon_{1}^{*}$ was defined in Remark 1 after Theorem 2.

One anticipates the most laborious task in computing $\epsilon_{0}$ to be the computation of the matrices $Q^{8}$ and $Q^{f}$. Rather than relying on the explicit form of these matrices revealed in Eqs. (17), (21) it is advisable to use instead an efficient numerical algorithm for directly solving the (linear) Liapunov matrix equations associated with $Q^{\prime}$ and $Q^{\prime}$. Because of this flexibility, the upper bound proposed here may prove less expensive to compute for large systems than that of [23], since the latter requires the evaluation of integrals on $[0, \infty)$ in which the matrix exponentials $e^{A_{0} t^{t}}$ and $e^{D t}$ appear. See Davison and Man [9] and Davison [10] for an algorithm for the numerical solution of Liapunov matrix equations. This algorithm converges in $O\left(n^{3}\right)$ steps, where $n$ is the number of rows (or columns) of the $n \times n$ unknown matrix $Q$.

A modest amount of numerical experimentation indicates that the upper bound obtained here fares respectably in relation to those of Zien, Chow and Khalil (see the Introduction). For systems in which $m=n=1$, so that $x$ and $y$ are scalars, however, Zien's result can be shown to be always less conservative than the one derived here. The formulae for this case are given below. The lower case letters $a, b, c, d$ represent the magnitudes of $A, B, C, D$ in (1), respectively, and $a_{0}:=\left|A_{0}\right|$. For this class of systems, Zien's upper bound [23] was found to be given by

$$
\epsilon_{0}=\frac{d^{2}}{2 b c} . \quad(Z i e n)
$$

The upper bound obtained using Theorem 3 above is

$$
\epsilon_{0}=\left\{\begin{array}{cc}
\frac{d^{2}}{2\left(a_{0} d+b c\right)} & \text { if } \frac{a_{0}}{b}<\frac{c}{d}, \text { or } \\
\frac{d^{2}}{\left(\left(a_{0} d\right)^{1 / 2}+(b c)^{1 / 2}\right)^{2}} & \text { if } \frac{a_{0}}{b}>\frac{c}{d} .
\end{array}\right.
$$

Some intermediate calculations used in the derivation of Eq. (26) are as follows. The value of $\epsilon_{2}$ 
is $d^{2} /\left(a_{0} d+c b\right)$. From (23) one computes $\bar{\alpha}=a_{0} / b$. Define $\alpha^{*}:=\left(\frac{a_{0} c}{b d}\right)^{1 / 2}$, so that $\bar{\alpha}<\alpha^{*}$ when $\left(a_{0} / b\right)<(c / d)$ and $\alpha^{*} \leq \bar{\alpha}$ otherwise. The upper bound $\epsilon_{\mathrm{o}}$ is $\epsilon_{1}(\bar{\alpha})$ in the former case and $\epsilon_{1}\left(\alpha^{*}\right)$ in the latter. Hence the estimate $(24)$ reduces to $\max _{0<\alpha<\bar{\alpha}} \epsilon_{1}(\alpha)$ in the case $n=m=1$.

\section{Conclusions}

The paper has presented a derivation of a new upper bound $\epsilon_{0}$ on the small paramecer $\epsilon$ of a singularly perturbed system (1) such that if Theorem 1 applies the asymptotic stability of (1) is ensured if $0<\epsilon<\epsilon_{0}$. The analysis consisted of applying the Brouwer fixed point theorem and Liapunov's direct method to obtain explicit estimates for well known results on time scale separation and on regular perturbation of stable linear systems. An expression for $\epsilon_{0}$ was then obtained by considering the implications of these estimates for the stability of fast and slow subsystems associated with system (1). Computation of the new upper bound involves only the numerical solution of two Liapunov matrix equations, for which efficient algorithms are available. The estimate of this paper was compared with Zien's original estimate [23] for the case of scalar fast and slow subsystems. For this case, the upper bound obtained here was shown to be the more conservative of the two, though the degree of conservativeness depends heavily on the specific system (see Eqs. (25), (26)) being studied. Further work is necessary before any general conclusions on the relative tightness of these and other existing estimates can be assessed for large systems, for which their use is clearly the only recourse.

\section{Acknowledgments}

The author is grateful to Prof. I.D. Mayergoyz, Prof. P.S. Krishnaprasad and Mr. J.-H. Fu of the University of Maryland for helpful comments. This work was supported in part by the National Science Foundation under Engineering Research Initiation Grant No. ECS-84-04275, and by the Power Engineering Program, Dept. of Elec. Eng., Univ. of Maryland, through grants from BG\&E, PEPCO, VEPCO, Bechtel and General Electric.

\section{References}

[1] E.H. Abed, "Multiparameter singular perturbation problems: Iterative expansions and asymptotic stability," Systems and Control Letters, (to appear).

[2] E.H. Abed, "Singularly perturbed Hopf bifurcation," IEEE Trans. Circuits and Systems, (to appear).

[3] R. Bellman, Introduction to Matrix Analysis, McGraw-Hill, New York (1960).

[4] M.S. Berger, Nonlinearity and Functional Analysis, Academic Press, New York (1977).

[5] R.W. Brockett, Finite Dimensional Linear Systems, John Wiley, New York (1970).

[6] K.W. Chang, “Singular perturbations of a general boundary value problem," SIAM J. Math. Anal. vol. 3, pp. 520-526 (1972).

[7] J.H. Chow, “Asymptotic stability of a class of non-linear singularly perturbed systems," $J$. Franklin Institute vol. 305, pp. 275-281 (1978).

[8] J.H. Chow, (Ed.), Time-Scale Modeling of Dynamic Networks with Applications to Power Systems, Springer-Verlag, Berlin (1982).

[9] E.J. Davison and F.T. Man, "The numerical solution of $A^{\prime} Q+Q A=-C$," IEEE Trans. Automatic Control vol. AC-13, pp. 448-449 (1968).

[10] E.J. Davison, "The numerical solution of $\dot{X}=A_{1} X+X A_{2}+D, X(0)=C, "$ IEEE Trans. Automatic Control vol. AC-20, pp. 566-567 (1975).

[11] C.A. Desoer and M.J. Shensa, "Networks with very small and very large parasitics: Natural frequencies and stability," Proc. IEEE vol. 58, pp. 1933-1938 (1970). 
[12] J.K. Hale, Ordinary Differential Equations, 2nd ed., R.E. Krieger Publishing Co., Malabar, FL (1980).

[13] S.H. Javid, "Uniform asymptotic stability of linear time-varying singularly perturbed systems," J. Franklin Institute vol. 305, pp. 27-37 (1978).

[14] T. Kato, Perturbation Theory for Linear Operators, Springer-Verlag, New York (1966).

[15] H.K. Khalil, "Asymptotic stability of nonlinear multiparameter singularly perturbed systems," Automatica vol. 17, pp. 797-804 (1981).

[16] P.V. Kokotovic, "A Riccati equation for block-diagonalization of ill-conditioned systems," IEEE Trans. Automatic Control vol. AC-20, pp. 812-814 (1975).

[17] P.V. Kokotovic, R.E. O'Malley, Jr., and P. Sannuti, "Singular perturbations and order reduction in control theory - An overview," Automatica vol. 12, pp. 123-132 (1976).

[18] P.V. Kokotovic, "Applications of singular perturbation techniques to control problems," SIAM Review vol. 26, pp. 501-550 (1984).

[19] V.R. Saksena, J. O'Reilly, and P.V. Kokotovic, "Singular perturbations and time-scale methods in control theory: Survey 1976-1983," Automatica vol. 20, pp. 273-294 (1984).

[20] N.R. Sandell, Jr., "Robust stability of systems with application to singular perturbations," Automatica vol. 15, pp. 467-470 (1979).

[21] G.W. Stewart, Introduction to Matrix Computations, Academic Press, New York (1973).

[22] M. Vidyasagar, Nonlinear Systems Analysis, Prentice-Hall, Englewood Cliffs, N.J. (1978).

[23] L. Zien, "An upper bound for the singular parameter in a stable, singularly perturbed system," J. Franklin Institute vol. 295, pp. 373-381 (1973). 